\title{
COMPORTAMENTOS DE RISCO PARA ACIDENTES DE TRÂNSITO: UM INQUÉRITO ENTRE ESTUDANTES DE MEDICINA NA REGIÄO SUL DO BRASIL
}

\author{
Selma Maffei de Andrade*, Darli Antonio Soares, Gabriel Pereira Braga, Jemima Herrero Moreira, \\ fábio Martins Nardo Botelho \\ Trabalho realizado no Centro de Ciências da Saúde, Departamento de Saúde Coletiva, \\ Universidade Estadual de Londrina, Londrina, PR
}

RESUMO - OBjETIVo. Os acidentes de trânsito são um dos principais responsáveis pela mortalidade de jovens brasileiros. Assim, 0 presente estudo objetivou verificar a prevalência de comportamentos de risco para esses acidentes em uma população jovem.

Mérodos. Questionários contendo questóes sobre comportamentos de risco para acidentes de trânsito foram respondidos por alunos da primeira à quarta série do curso de Medicina da Universidade Estadual de Londrina, em outubro e novembro de 2000. Para a análise das diferenças de comportamentos entre os sexos usou-se o teste do qui quadrado, com um nível de significância de $5 \%$.

RESULTADOS. Em comparação às jovens, os rapazes relataram maior freqüência, estatisticamente significativa, para: locomoção como condutor de carro, possuir carteira de habilitação, ter aprendido a dirigir automóvel com 16 anos ou menos e ter ingerido bebida alcoólica previamente à direção de veículo nos últimos 30 dias. Entre os condutores regulares de automóvel, observou-se também menor proporção de comportamentos desejáveis entre os alunos do sexo masculino. Falta de atenção $(59,3 \%)$, desrespeito à sinalização $(33,5 \%)$ e excesso de velocidade $(22,5 \%)$ foram os fatores mais citados como determinantes para a ocorrência do último acidente, sem diferença entre os sexos.

Conclusöes. Os resultados indicam a necessidade de medidas visando à redução de fatores que favorecem a ocorrência de acidentes de trânsito entre esses jovens, especialmente os do sexo masculino.

UNITERMOS: Acidentes de trânsito. Fatores de risco. Jovens.

\section{INTRODUÇÃO}

Apesar da redução das taxas de mortalidade e da gravidade dos acidentes de trânsito no Brasil emanos recentes, a situação ainda inspira grande preocupaçã ${ }^{\prime, 2}$, a ponto de merecer atenção especial do Ministério da Saúde, que propôs, recentemente, um programa de redução da sua morbimortalidade ${ }^{3}$.

Os acidentes e violências - denominados causas externas de morbidade e mortalidade, de acordo com a Classificação Internacional de Doenças ${ }^{4}$-correspondemà segunda principal causa de óbito da população brasileira em geral. No entanto, na faixa etária entre os $5 \mathrm{e}$ os 39 anos, esses eventos representam a primeira causa de morte, sendo os homicídios e os acidentes de trânsito os grandes responsáveis por essa mortalidade ${ }^{5}$.

\footnotetext{
* Correspondência:

Universidade Estadual de Londrina

Centro de Ciências da Saúde

Departamento de Saúde Coletiva

Rua Robert Koch, 60 - Vila Operária - CeP: 86.038-440

Londrina - PR - Brasil - Telefone: (43) 3371-2274

Fax: (43) 3323-2808-semaffei@sercomtel.com.br
}

Esse expressivo número de acidentes representa significativo impacto na economia do país, não só pelos altos custos com atendimento e internação das vítimas ${ }^{6}$, como $^{2}$ também por ser responsável pela morte de expressiva parceladapopulaçãoeconomicamente ativa. Além disso, a prematuridade e, em geral, o modo repentino como essas mortes ocorrem geram enorme sofrimento e transtornos psicossociais às famílias envolvidas.

Alguns fatores têm sido destacados na literatura médica como determinantes da origeme dagravidade dos acidentes de trânsito. Dentre esses, são freqüentemente citados a idade, o gênero, as condições socioeconômicas, o desrespeito à legislação de trânsito-especialmente o abuso de velocidade e o consumo de bebidas alcoólicas previamente à direção de veículos automotores-, associados, em geral, a uma inadequada fiscalização do trânsito ${ }^{7-9}$.

A alta incidência de vítimas jovens, em diversas sociedades, vem sendo relacionada à falta de experiência na condução de veículos, além de características próprias da juventude, como a impulsividade ea necessidade de auto- afirmação perante o grupo de pares. Como agravante, os jovens, geralmente, consomem mais freqüentemente bebidas alcoólicas e drogas do que os adultos, bem como tendem a exceder mais os limites de velocidade e a desrespeitar outras normas de segurança no trânsito, o que, sabidamente, aumenta as chances de ocorrência de acidentes ${ }^{7-11}$.

Considerando que a população jovem éa mais vulnerável à ocorrência de acidentes de trânsito, como diversos estudos denotam, faz-se importante estudar a frequiência de determinados comportamentos de risco para tais acidentes nesse segmento da população. Enquanto países como os Estados Unidos da América contam com dados de coleta periódica sobre esses comportamentos entre jovens, permitindo seu monitoramento, no Brasil são raros esses tipos de estudo' ${ }^{12}$. $O$ presente trabalho, portanto, teve como finalidade verificar a freqüência de comportamentos que podem contribuir para a ocorrência ou para a gravidade de acidentes de trânsito entre jovens universitários de uma instituição de ensino público no Sul do Brasil. 


\section{Métodos}

A população de estudo foi composta por alunos do curso de Medicina da Universidade Estadual de Londrina - UEL, que, durante 0 ano de 2000 , cursavam da primeira à quarta série. Onze alunos do segundo ano foram excluídos, pois participavam na condução da presente pesquisa, resultando em um total de 309 alunos a serem incluídos ( 80 em cada ano cursado, exceto os II do segundo ano).

O instrumento para a coleta de dados foi um questionário, auto-respondido de maneira anônima, com perguntas abordando assuntos relacionados a fatores de risco e à ocorrência de acidentes de trânsito. $O$ mesmo foi planejado tendo como parâmetro o desenvolvido e aplicado na pesquisa "Youth Risk Behavior Survey", nos Estados Unidos da América' ${ }^{13}$. Esse instrumento tem como propósito monitorar uma série de comportamentos de jovens norte-americanos que possam afetar sua saúde. No presente estudo, foram utilizadas apenas as questões referentes a comportamentos no trânsito, acrescidas de outras pertinentes ao levantamento de dados relacionados à ocorrência de acidentes e outros comportamentos que sabidamente ocorrem na realidade brasileira. $O$ protocolo de pesquisa, incluindo seu instrumento, foi submetido ao Comitê de Ética em Pesquisa da Universidade Estadual de Londrina, sendo aprovado.

Primeiramente, o questionário foi respondido por alunos de ambos os sexos de outros cursos da área da saúde da UEL (Enfermagem, Farmácia, Odontologia e Fisioterapia), objetivando verificar a plena compreensão das questões. Algumas pequenas alterações foram realizadas, especialmente nas opções de resposta de algumas perguntas. Em seguida, o questionário reformulado foi aplicado em sala de aula aos estudantes das primeiras quatro séries do curso de Medicina, durante os meses de outubro e novembro de 2000, após obter autorização do professor responsável. Foi feita uma explanação por um dos autores sobre a importância e os objetivos deste trabalho; solicitado o consentimento livre e esclarecido, de forma verbal e escrita, e enfatizada a necessidade de que as respostas dadas correspondessem ao máximo à realidade. Em geral, o questionário era respondido entre 20 a 30 minutos.

Posteriormente, os dados obtidos foram processados e analisados com o auxílio do programa Epi Info versão $6.04 d^{14}$. Para a análise

\section{Figura I-Meios de transporte mais utilizados entre estudantes de medicina segundo o sexo. Londrina, 2000}

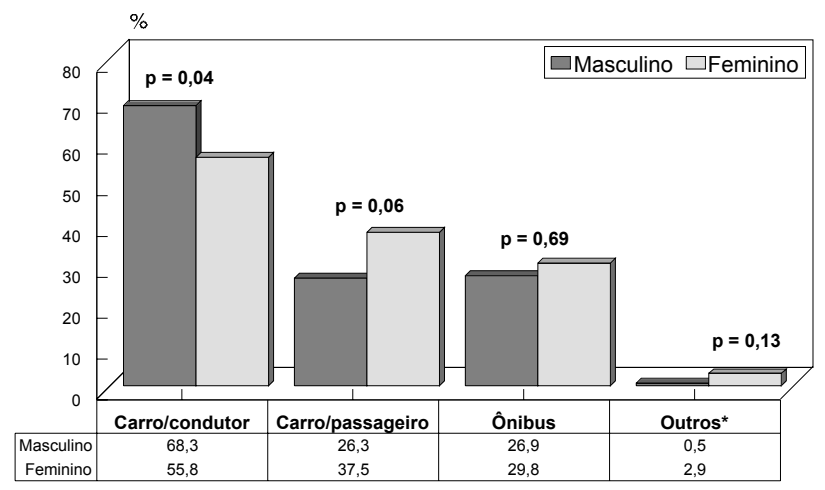

Meio de transporte

*Bicicleta/motocicleta

OBS.: Permitidas múltiplas respostas, se dois ou mais meios de transporte fossem igualmente utilizados

Tabela I - Características e comportamentos de risco no trânsito entre estudantes de Medicina segundo o sexo. Londrina, 2000

\begin{tabular}{|c|c|c|c|c|c|}
\hline \multirow{2}{*}{ Características/comportamentos } & \multicolumn{2}{|c|}{ Masculino $(\mathrm{N}=186)$} & \multicolumn{2}{|c|}{ Feminino $(N=104)$} & \multirow{2}{*}{$\underline{p-v a l o r}$} \\
\hline & $\mathbf{N}$ & $(\%)$ & $\mathbf{N}$ & $\%$ & \\
\hline Possuihabilitaçãopara dirigir carro & 168 & 90,3 & 82 & 78,8 & 0,01 \\
\hline Aprendeua dirigir com $\leq 16$ anos & 118 & 63,4 & 35 & 33,7 & $<0,001$ \\
\hline $\begin{array}{l}\text { Ingestão de bebidaalcoólica edireçãa } \\
\text { deveículo* }\end{array}$ & 83 & 44,6 & 21 & 20,2 & $<0,001$ \\
\hline $\begin{array}{l}\text { Passageiroem veículocom condutor que } \\
\text { ingeriubebidaalcoólica* }\end{array}$ & 73 & 39,2 & 46 & 44,2 & 0,48 \\
\hline $\begin{array}{l}\text { Como condutor, usa cinto de segurança } \\
\text { sempre** }\end{array}$ & 155 & 89,6 & 75 & 88,2 & 0,91 \\
\hline $\begin{array}{l}\text { Como passageiro do banco dianteiro, } \\
\text { usa cinto de segurança sempre**** }\end{array}$ & 159 & 86,9 & 86 & 83,5 & 0,54 \\
\hline $\begin{array}{l}\text { Como passageiro do banco traseiro, } \\
\text { usa cinto de segurança sempre }{ }^{* * *}\end{array}$ & 14 & 7,7 & 5 & 4,9 & 0,53 \\
\hline Participaçãoem "racha" & 29 & 15,6 & 10 & 9,6 & 0,21 \\
\hline Envolvimentoemacidentedetrânsito & 122 & 65,6 & 60 & 57,7 & 0,23 \\
\hline
\end{tabular}

*nosúltimos 30 dias

**\% calculado excluindo os que não dirigem veículo ou que não são passageiros de carros

das diferenças nas freqüências de comportamento entre os sexos foi utilizado o teste do qui quadrado, com um nível de significância de $5 \%$.

\section{Resultados}

Um total de 290 alunos respondeu 0 questionário, correspondendo a $93,9 \%$ da população a ser pesquisada. As perdas decorreram exclusivamente por ausência de alunos no dia da aplicação do questionário, não havendo recusa. Entre os alunos que responderam, a idade variou de 17 a 27 anos, com média e mediana de $2 \mathrm{I}$ anos (desvio padrão de
I,7 anos). A moda, de 22 anos, correspondeu, também, ao terceiro quartil. A maioria dos alunos era do sexo masculino $(64,1 \%)$.

Carro foi o meio de transporte citado como o mais utilizado pelos estudantes, especialmente na sua condução por alunos do sexo masculino, com diferença estatisticamente significativa entre os sexos (Figura I). Diferença significativa também foi observada em relação ao sexo quanto à posse de carteira de habilitação para conduzir veículo automotor, pois $90,3 \%$ dos rapazes referiram possuí-la contra apenas $78,8 \%$ das jovens (Tabela I). 
Com relação à idade em que aprenderam a dirigir automóvel, houve predominância do sexo masculino aprendendo a dirigir antes dos 18 anos, idade mínima, pela legislação brasileira, para tal aprendizado. Comparando, ainda, o aprendizado com idade de 16 anos ou menos, observa-se diferença significativa ( $p<0,001)$ entre os sexos, com $63,4 \%$ dos rapazes aprendendo a dirigir precocemente, em comparação a $33,7 \%$ das jovens (Tabela I). Familiares, especialmente o pai $(60,7 \%)$, foram os principais responsáveis pelo ensino da direção de automóvel.

Aingestão de bebida alcoólica previamenteà condução de veículo automotor foi relatada por quase a metade dos universitários do sexo masculino, em comparação a cerca de $20 \%$ das jovens, com diferença significativa (Tabela I). Em contrapartida, as mulheres relataram ser mais freqüentemente passageiras em veículo cujo condutor havia ingerido bebida alcoólica $(44,2 \%)$, embora sem diferença estatística entre os sexos.

Quanto ao uso do cinto de segurança, não houve diferença estatística entre os sexos em quaisquer posições ocupadas no automóvel. Chama a atenção, no entanto, o fato de ser baixa a freqüência de usuários desse equipamento de segurança quando o transporte ocorre como passageiro do banco traseiro do carro, conforme mostra a Tabela I.

Não houve diferença significativa entre os sexos, também, no relato de participação em disputaautomobilística em via pública("racha"), embora os jovens do sexo masculino tenham apresentado maior freqüência desse comportamento de risco (Tabela I). Para esse comportamento não se argüiu se os jovens participaram como condutores ou passageiros.

O envolvimento em acidente (como condutor ou passageiro) foi relatado por número expressivo de universitários: cerca de dois terços dos rapazes e mais da metade das jovens, embora sem diferença significativa, conforme mostra a Tabela I. Cerca de 12\% dos jovens relataram ter se envolvido em três ou mais acidentes, também sem diferença estatística entre os sexos (dados não mostrados).

Entre os que relataram participação em acidente, os principais fatores percebidos como contribuintes para a ocorrência ou gravidade do último acidente em que estiveram envolvidos foram falta de aten-

Figura 2 - Fatores que contribuíram para a ocorrência ou gravidade do último acidente de trânsito segundo a percepção de estudantes de Medicina. Londrina, 2000

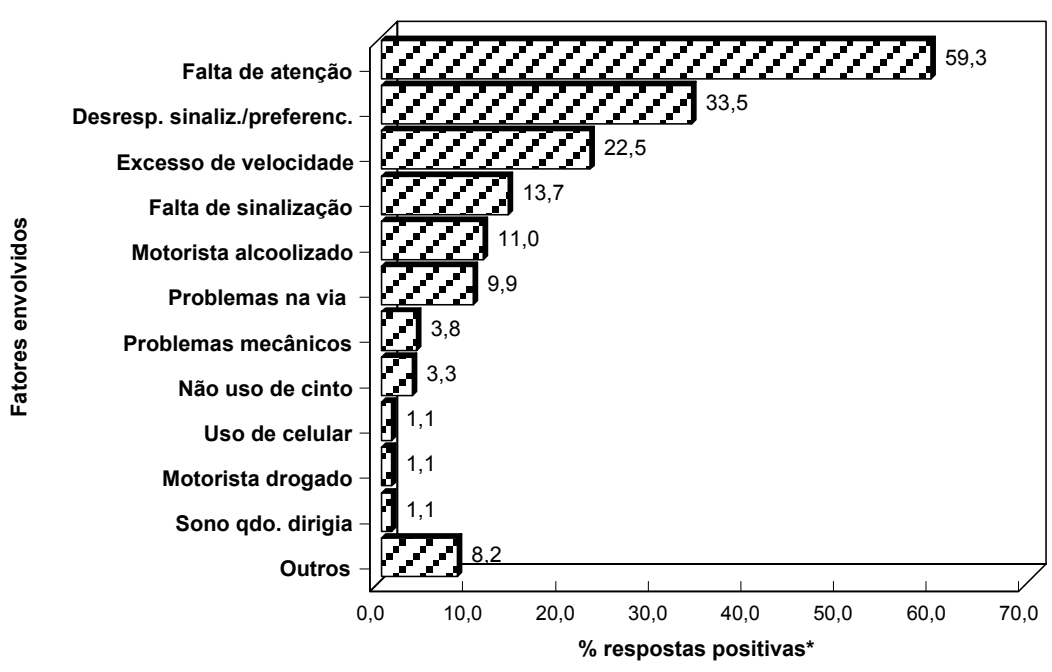

* Admite múltiplas respostas. Percentual calculado entre os alunos que se envolvem em acidentes

Tabela 2-Comportamentos de risco no trânsito entre estudantes de Medicina que dirigem regularmente automóvel segundo o sexo. Londrina, 2000

\begin{tabular}{|c|c|c|c|c|c|}
\hline \multirow[t]{2}{*}{ Comportamentos } & \multicolumn{2}{|c|}{ Masculino $(\mathrm{N}=127)$} & \multicolumn{2}{|c|}{ Feminino $(N=58)$} & \multirow[t]{2}{*}{$\mathrm{p}$-valor } \\
\hline & $\mathbf{N}$ & $(\%)$ & $\mathbf{N}$ & $\%$ & \\
\hline Sempre respeita olimite develocidade & 21 & 16,5 & 10 & 17,2 & 0,93 \\
\hline Semprerespeitasemáforo & 59 & 46,5 & 37 & 63,8 & 0,04 \\
\hline Nuncafazultrapassagem proibida & 52 & 40,9 & 38 & 65,5 & $<0,01$ \\
\hline Nuncafazconversãoilegal & 40 & 31,5 & 27 & 46,6 & 0,07 \\
\hline Sempre respeita afaixa de pedestre & 96 & 75,6 & 48 & 82,8 & 0,37 \\
\hline Nuncafala ao celular quando dirige* & 32 & 41,6 & 23 & 48,9 & 0,54 \\
\hline Nãoingeriubebidaalcoólica antes de dirigir** & 59 & 46,5 & 40 & 69,0 & $<0,01$ \\
\hline
\end{tabular}

*\% calculado em relação aos condutores que possuem celular

*** nosúltimos 30 dias

ção $(59,3 \%)$, desrespeito à sinalização ou preferencial $(33,5 \%)$ e excesso de velocidade (22,5\%). A ingestão prévia de bebida alcoólica pelo condutor aparece em quinto lugar, com II,0\% (Figura 2).

Entre os alunos que referiram ser costumeiros condutores de automóveis (127 do sexo masculino e 58 do feminino) houve diferenças significativas, entre os sexos, para comporta- mentos desejáveis no trânsito, como "nunca faz ultrapassagem proibida", "sempre respeita 0 semáforo" e "não ingeriu bebida alcoólica antes dedirigirnosúltimos 30 dias" (Tabela 2), menos freqüentes entre os rapazes. Nota-se, nessa mesma tabela, que esses comportamentos esperados no trânsito não apresentam alta freqüência, sendo o respeito ao limite de velocidade o menos observado por alunos e alunas. 


\section{Discussão}

Por se tratar de uma população composta por universitários de um curso altamente concorrido, de poder aquisitivo relativamente alto, e pelo fato de o carro ser o principal meio de transporte, os estudantes deste estudo não representam a população jovem brasileira, que, em geral, tem menos acesso a esse meio de transporte. $O$ conhecimento das peculiaridades de cada população, no entanto, é fundamental para que as estratégias de prevenção possam ter um impacto mais efetivo ${ }^{15}$. Por outro lado, os achados do presente trabalho podem contribuir para uma reflexão precoce desses futuros profissionais de saúde sobre a magnitude do problema e da sua responsabilidade, quando profissionais, na prevenção dos acidentes de trânsito e não apenas no atendimento às vítimas desses eventos.

Um outro ponto a ser destacado é que estudos que se baseiam em questionários auto-respondidos têm limitações, geralmente levando a menores taxas de relatos de comportamentos socialmente condenáveis. Essa limitação tem sido atenuada, como no presente estudo, pela utilização de questionários anônimos, de preenchimento voluntário e com garantia verbal e escrita, por parte dos pesquisadores, quanto ao caráter confidencial das informações ${ }^{\prime 2}$.

Considerando essas limitações, a proporção de relatos de envolvimento em acidentes de trânsito, no presente estudo, foi alta e não houve diferença estatística entre os sexos, embora não se tenha questionado se os alunos estavam na posição de condutor ou de passageiro do veículo no momento do acidente e, tampouco, a sua gravidade. Tendo em vista as características da população de estudo, predominantemente usuária de automóveis, esse achado pode ser explicado pelos resultados encontrados em outra investigação ${ }^{16}$ que, analisando diferentes tipos de vítimas de acidentes de trânsito, encontra menores diferenças de sexo entre os ocupantes desse meio de locomoção (I,6 homem para cada mulher), em comparação, por exemplo, a cerca de cinco homens para cada mulher ciclista. Outra possível explicação para a inexistência de diferença de envolvimento em acidentes, entre os sexos, destacada por Valent et al. ${ }^{17}$, é que as mulheres tendem a se envolver mais em acidentes de pequena monta, resultando geralmente em ferimentos leves, enquanto os homens se envolvem mais freqüentemente em acidentes graves, com maior risco de morte. Possivelmente, esses distintos tipos de desfechos se devam às diferenças na exposição ao trânsito, na habilidade/experiência na direção de veículos e nas decisões em correr riscos. No presente trabalho, confirmou-se a tendência de os rapazes terem maior tempo de exposição ao trânsito, considerando o tempo de aprendizado e a posse da carteira de motorista.

Além dessa maior exposição masculina ao trânsito, foram também observadas outras diferenças quanto à freqüência de relatos de comportamentos de risco entre os jovens estudados, de acordo com o sexo. De modo geral, os rapazes relataram mais freqüentemente serem condutores de carro, possuírem habilitação para dirigir, terem aprendido a dirigir com menor idade e terem ingerido bebida alcoólica previamente à condução de carro nos últimos 30 dias. Entre os condutores habituais de automóvel, também foi observada menor freqüência de comportamentos desejáveis entre os jovens do sexo masculino, como nunca fazer ultrapassagem proibida, sempre respeitar o semáforo e não ter ingerido bebida alcoólica antes de dirigir carro nos últimos 30 dias. Estes resultados são consistentes na maioria dos estudos que relatam que os jovens do sexo masculino tendem a assumir comportamentos de risco à saúde mais freqüentemente do que as do sexo feminino e são as principais vítimas de acidentes de trânsito ${ }^{5,16,18-20}$. No entanto, deve-se destacar a grande parcela de mulheres que participa menos ativamente em atividades de risco, como, por exemplo, quando são passageiras em veículo cujo condutor ingeriu bebida alcoólica.
O fato de muitos terem aprendido a dirigir antes da idade mínima legal reflete insuficiente conscientização quanto aos riscos de se ter um menor ao volante, que é confirmada pelo fato de a maioria dos entrevistados ter aprendido a dirigir com os próprios familiares, evidenciando a necessidade de desenvolvimento de estratégias de mudanças deste tipo de comportamento ${ }^{21}$. Além disso, muitos declararam já ter participado de "rachas", prática extremamente perigosa, tanto para os que a realizam como para os demais usuários da via pública.

O uso do cinto de segurança foi relatado por mais de $80 \%$ dos estudantes quando viajam na posição de condutores ou passageiros do banco dianteiro do carro, valor superior ao detectado em outro estudo na mesma cidade entre vítimas de acidentes de carro $(62,6 \%)^{22}$. Entretanto, a proporção de usuários desse dispositivo de segurança quando no banco traseiro do veículo é ínfima, o que talvez seja explicado pelo fato de a maioria dos carros brasileiros ainda contar com cintos traseiros abdominais, que muitas vezes ficam camuflados sob o banco do automóvel, além da baixa fiscalização sobre seu uso. $O$ cinto de segurança é considerado 0 dispositivo mais eficaz em termos de redução da gravidade do trauma em acidentes de automóvel ${ }^{17,18}$ e é o equipamento de segurança mais disponível nos veículos que circulam atualmente no Brasil. Possivelmente, a regulamentação da produção de veículos com cintos traseiros de três pontos e o estabelecimento de medidas, pela indústria automobilística, visando alertar o passageiro sobre a falta do cinto, pudessem aumentar sensivelmente sua utilização por usuários do banco traseiro do veículo.

A percepção dos estudantes em relação aos principais fatores que contribuíram para seu último acidente de trânsito, tais como desrespeito à sinalização, excesso de velocidade e motorista alcoolizado, indica que medidas de estímulo à direção defensiva, aumento da fiscalização, implantação de mecanismos de redução da velocidade dos veículos e melhoria da sinalização do trânsito poderiam, provavelmen- 
te, surtir algum efeito na redução desses eventos entre os jovens estudados ${ }^{1-3,16-20}$. Com relação à falta de atenção, principal motivo apontado pelos estudantes, não há estudos brasileiros que avaliem as causas fundamentais de distração no trânsito, o que poderia subsidiar algumas medidas preventivas ${ }^{23}$.

Finalmente, há que se considerar que 0 comportamento dos jovens estudados, os quais são predominantemente condutores de automóvel, pode colocar outros usuários da via pública, mais vulneráveis, como os pedestres e ciclistas, sob maior risco de trauma grave em acidentes decorrentes de seus comportamentos inadequados no trânsito. Embora as medidas de prevenção de acidentes de trânsito mais eficazes sejam aquelas voltadas às modificações do meio ambiente ${ }^{24,25}$ (incluindo a indústria automobilística e legislação eficiente), não se pode, atualmente, com as atuais taxas de morbimortalidade por acidentes de trânsito, prescindir de medidas que visem, também, modificações positivas nos comportamentos dos indivíduos.

\section{Conclusões}

Foram observados diversos comportamentos de risco para acidentes de trânsito entre os jovens universitários deste estudo, especialmente entre os do sexo masculino. Medidas que visem modificálos deveriam ser colocadas em prática urgentemente, no sentido de diminuir riscos a si próprios e a outros usuários da via pública e aumentar a conscientização sobre a questão da importância da segurança no trânsito.

\section{Agradecimentos}

Aos acadêmicos do curso de Medicina da Universidade Estadual de Londrina que participaram da coleta de dados e da sistematização dos primeiros resultados: Adriana Koga Bertolino, Anselmo Nunes Duarte Junior, GuiIherme Genta dos Santos, Karine Tosta, Leonardo Oba, Paulo Muller Ramos, Raíssa Medeiros de Florenço, Viviane Christina Siena. Aos demais alunos e professores do curso que permitiram a realização do trabalho. $\mathrm{Ao} \mathrm{CNPq}$, pela concessão de bolsas de iniciação científica.

\section{SUMMARY}

RISKY BEHAVIOR FOR TRAFFIC ACCIDENTS: A SURVEY AMONG MEDICAL STUDENTS IN SOUTHERN BraZiL

BACKGROUND. Traffic accidents are one of the most frequent cause of mortality among Brazilian youths. Therefore, this study aimed at detecting the prevalence of risk factors for such accidents in a young population.

METHODS. Questionnaires containing questions about risky behavior for traffic accidents were answered by students from the first to the fourth year of the Medical course of the Sate University of Londrina, Paraná State, Brazil, during October and November, 2000. The chi-square test was used to detect differences between the sexes, with a $5 \%$ significance level.

RESULTS. Compared to women, male students reported a statistically significant higher frequency of transportation as a car driver, owing a driver license, to have learnt to drive a car under the age of 16 and to have drunk alcoholic beverages before driving a vehicle during the last 30 days. Among regular drivers, a lower proportion of desirable behavior was also noted among male students. Lack of attention (59.3\%), disregarding traffic signals (33.5\%) and speeding $(22.5 \%)$ were the main factors cited as determinants for the occurrence of the last accident, with no gender difference.

Conclusions. Theseresultspoint out for theneed to implement measures in order to reduce factors that favors the occurrence of traffic accidents among these youths, especially among males. [Rev Assoc Med Bras 2003; 49(4): 439-44]

KEY wORDS: Traffic Accidents. Risk Factors. Youth.

\section{REFERÊNCIAS}

I. Queiroz MS, Oliveira PCP. Acidentes de trânsito: uma visão qualitativa no município de Campinas, São Paulo, Brasil. Cad Saúde Pública 2002; I8: I $179-87$.

2. Farage L, Colares VS, Capp Neto M, Moraes MC, Barbosa MC, Branco Júnior JA. As medidas de segurança no trânsito e a morbimortalidade intra-hospitalar por traumatismo craniencefálico no Distrito Federal. Rev Assoc Med Bras 2002; 48: 163-6.

3. Ministério da Saúde. Secretaria de Políticas de Saúde. Programa de redução da morbimortalidade por acidentes de trânsito: mobilizando a sociedade e promovendo a saúde. Rev Saúde Pública 2002; 36: I | 4-7.
4. Organização Mundial da Saúde. Manual de classificação estatística internacional de doenças e problemas relacionados à saúde. $10^{\mathrm{a}}$ rev. São Paulo: Centro Colaborador da OMS para Classificação de Doenças em Português; 1993. v.I.

5. Mello Jorge MHP, Gawryszewski VP, Latorre MRDO. Acidentes e violências no Brasil. I Análise dos dados de mortalidade. Rev Saúde Pública 1997; 3 I (Supl 4):5-25.

6. lunes RF. Acidentes e violências no Brasil. III. Impacto econômico das causas externas no Brasil: um esforço de mensuração. Rev Saúde Pública 1997; 3 I (Supl4):438-46.

7. Shibata A, Fukuda K. Risk factors of fatality in motor vehicle traffic accidents. Accid Anal Prev 1994; 26:391-7.

8. Lourens PF, Vissers JAMM, Jessurun M. Annual mileage, driving violations and accident involvement in relation to drivers' sex, age and level of education. Accid Anal Prev 1999; 31:593-7.

9. Hasselberg M, Laflamme L, Weitoft GR. Socioeconomic differences in road traffic injuries during childhood and youth: a closer look at different kinds of road user. J Epidemiol Community Health 200 I ; 55:858-62.

10. EverettSA, Lowry R, Cohen LR, Dellinger AM. Unsafe motor vehicle practices among substance using college students. Accid Anal Prev 1999; 31 :667-73.

II. Longo MC, Hunter CE, Lokan RJ, White JM, White MA. The prevalence of alcohol, cannabinoids, benzodiazepines and stimulants amongst injured drivers and their role in driver culpability. Part I: the prevalence of drug use in drivers, and characteristics of the drug-positive group. Accid Anal Prev 2000; 32:6 I 3-22.

12. Carlini-Cotrim B, Gazal-Carvalho C, Gouveia $\mathrm{N}$. Comportamentos de saúde entre jovens estudantes das redes pública e privada da área metropolitana do Estado de São Paulo. Rev Saúde Pública 2000; 34:636-45.

13. Centers for Disease Control and Prevention (CDC). 1999 National School-based youth risk behavior survey [online]. Disponível em: URL: http://www.cdc.gov/nccdphp/dashyrbs /data/ 1999/yrbs 1999.pdf. Acessado em: 6 maio 2000.

14. Dean AG, Dean JA, Coulombier D, Brendel KA, Smith DC, Burton AH et al. Epi Info, version 6: a word-processing, database, and statistics program for public health on IBMcompatible microcomputers. Atlanta, Georgia, USA: Centers for Disease Control and Prevention; 1995.

I5. Birolini D. Como anda a epidemia do trauma? Rev Ass Med Bras 200I ; 47:3.

16. Andrade SM, MelloJorge MHP. Características das vítimas por acidentes de transporte terrestre em município da Região Sul do Brasil. Rev Saúde Pública 2000; 34: 149-56.

17. Valent F, Schiava F, Savonitto C, Gallo T, Brusaferro S, Barbone F. Risk factors for fatal road traffic accidents in Udine, Italy. Accid Anal Prev 2002; 34:7I-84. 
18. Posada J, Ben-Michael E, Herman A, Kahan E, Richter E. Death and injury from motor vehicle crashes in Colombia. Rev Panam Salud Pública 2000; 7:88-91.

19. Mayrose J, Jehle DV. An analysis of race and demographic factors among motor vehicle fatalities. J Trauma 2002; 52:752-5.

20. Hingson R, Heeren T, Levenson S, Jamanka A, Voas R. Age of drinking onset, driving after drinking, and involvement in alcohol related motor-vehicle crashes. Accid Anal Prev 2002; 34:85-92.
21. Dellatorre MCC. O trânsito e seus novos centauros [dissertação]. Londrina: Centro de Ciências da Saúde, Universidade Estadual de Londrina; 1997.

22. Liberatti CLB, AndradeSM, Soares DA. Thenew brazilian traffic code and some characteristics of victims in southern Brazil. Inj Prev 200 I; 7:190-3.

23. Stutts JC, Reinfurt DW, Rodgman EA. The role of driver distraction in crashes: an analysis of 1995-1999 Crashworthiness Data System Data. Annu Proc Assoc Adv Automot Med 200I; 45:287-30I.
24. Stevenson M. Childhood pedestrian injuries: what changes to the road environment achieve? Aust NZ J Public Health 1997; 21:33-7.

25. Abassi K. War on the roads. BMJ 2002; 324:1107-8.

Artigo recebido: 10/02/2003

Aceito para publicação: 05/08/2003

\section{IMAGEM EM MEDICINA}

Participe da nova seção da Ramb. É um espaço aberto ao leitor, que poderá enviar material de interesse educativo como fotos, ilustrações e exames, acrescido de três linhas explicativas contendo ainda nome do autor e serviço onde foi realizado. O material poderá ser enviado para a

Rua São Carlos do Pinhal, 324 - Cep 01333-903

S. Paulo - SP - E-mail: ramb @ amb.org.br 